\title{
Diferentes escalas de reprodução e migração em um sistema presa-predador com estrutura espacial
}

\author{
Carina L. Andrade, Jacques A. L. da Silva, \\ Instituto de Matemática, UFRGS, \\ 90040-060, Porto Alegre, RS \\ E-mail: carinalandrade@gmail.com, jaqx@mat.ufrgs.br,
}

Resumo: $O$ objetivo principal deste trabalho é analisar a presença de duas escalas distintas de tempo em um modelo metapopulacional do tipo presa-predador, discreto no tempo e no espaço. Inicialmente, analisamos duas situaçôes: presas com dinâmica vital mais rápida do que predadores e vice-versa. Verificou-se que esta consideração de mais de uma escala de tempo para a reprodução das espécies modifica a região de estabilidade das soluções de equilíbrio de coexistência, altera as densidades das duas espécies e o tipo de soluções observadas. A seguir, considera-se uma metapopulação, ou seja, as duas espécies distribuem-se em sítios e ocorre migração. Essa migração ocorrerá de forma coerente com a escala de reprodução. A consideração de estrutura espacial também altera as predições do modelo.

Palavras-chave: Diferentes escalas de tempo, Metapopulação, Sistema presa-predador

\section{Introdução}

Em sistemas biológicos pode ocorrer que uma das espécies se reproduza em intervalos de tempo diferentes daqueles da outra espécie. Na natureza encontramos muitos exemplos de interações nas quais uma espécie reproduz-se em intervalos de tempo menores do que a outra: podemos citar joaninhas predando afídeos [2], doninhas atacando pequenos roedores [6], controle da cochonilha vermelha (Aonidiella auranti) pelo parasitóide Aphytis melinus [4].

Kindlmann e Dixon [2] argumentam que o efeito na densidade populacional de presas é inversamente relacionado ao tempo de desenvolvimento do predador. Em termos biológicos, essa teoria prediz que, dada uma espécie de presas e dois predadores diferindo apenas nos tempos de desenvolvimento, o predador com tempo de desenvolvimento mais longo terá menos efeitos na abundância de presas. Kindlmann et al. [3] relatam testes realizados em campo que trazem resultados de acordo com a hipótese descrita acima.

Assim, neste trabalho propomos um modelo presa-predador discreto com crescimento das presas dependendo da densidade em que a reprodução das espécies ocorre em escalas diferentes de tempo. Analisamos duas situações: presas possuem dinâmica mais rápida do que predadores e vice-versa.

Os trabalhos de Rodrigues et al. [7] e Andrade [1] analisam um modelo presa-predador discreto com dinâmica da presa mais rápida que a dinâmica do predador. É observado que as densidades das presas diminuem quanto maior a diferença entre as escalas de reprodução das duas espécies, o que não está de acordo com as conclusões e testes em campo de Kindlmann e Dixon [3]. Neste trabalho será proposto um modelo diferente, em que a predação ocorre de acordo com a média das densidades de presas nas gerações intermediárias e não de acordo com a soma das densidades nessas gerações como é feito em [7] e [1].

Os estudos de dinâmicas populacionais têm cada vez mais levado em conta a distribuição espacial das espécies. Rohani e Ruxton [8] discutem sistemas com mais de uma espécie e chegam a resultados de que a extrema assimetria nas frações de dispersão entre as duas espécies, pode 


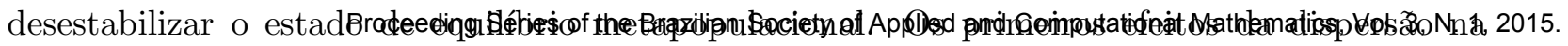
estabilidade de um modelo foram mencionados no trabalho de Turing [9].

Em [1] temos ainda a proposta de um modelo presa-predador com estrutura espacial e dinâmica vital das presas mais rápida que a dos predadores. A movimentação de ambas espécies ocorre em escala rápida, ou seja, a cada gerãção em que temos a reprodução das presas, as duas espécies migram. Neste trabalho, propomos um modelo em que a migração é coerente com a escala de reprodução das espécies e a estrutura espacial será considerada para os dois casos: presa rápida $\mathrm{x}$ predador lento e presa lenta x predador rápido.

\section{Formulação do Modelo}

Partiremos de um modelo presa-predador, proposto por Neubert e Kot [5], considerando uma única escala de tempo:

$$
\left\{\begin{array}{l}
h_{t+1}=h_{t} \exp \left(r\left(1-h_{t}-p_{t}\right)\right), \\
p_{t+1}=\gamma p_{t}+d h_{t} p_{t} .
\end{array}\right.
$$

onde, $h_{t}$ e $p_{t}$ são, respectivamente, as densidades adimensionais de presas e predadores na geração $t$ e $d, r$ e $\gamma$ são parâmetros adimensionais positivos.

Os pontos de equilíbrio do sistema (1) são:

$$
\left(h_{0}, p_{0}\right)=(0,0), \quad\left(h_{1}, p_{1}\right)=(1,0) \quad e \quad\left(h^{*}, p^{*}\right)=\left(\frac{1-\gamma}{d}, 1-\frac{1-\gamma}{d}\right) .
$$

$\left(h_{0}, p_{0}\right)$ é o ponto de equilíbrio trivial, no qual temos extinção de ambas espécies. $\left(h_{1}, p_{1}\right)$ representa extinção da população de predadores, com a densidade de presas igual à capacidade suporte do meio. Finalmente, $\left(h^{*}, p^{*}\right)$ é o único ponto de equilíbrio que apresenta coexistência de presas e predadores, porém este ponto só é viável biologicamente quando $d>1-\gamma$.

A análise de estabilidade do sistema (1) indica que o ponto de equilíbrio $\left(h_{0}, p_{0}\right)$ é sempre instável para quaisquer $r>0$ e $\gamma>0$; o ponto de equilíbrio $\left(h_{1}, p_{1}\right)$ é estável se $d<1-\gamma$ e $r<2$; e as condições para que tenhamos a estabilidade local do ponto de equilíbrio $\left(h^{*}, p^{*}\right)$ são:

$$
1-\gamma<d<2-\gamma \quad \text { e } \quad r<\frac{4 d}{(3-d-\gamma)(1-\gamma)}
$$

A Figura 1 mostra as regiões de estabilidade dos pontos de equilíbrio $\left(h_{1}, p_{1}\right)$ e $\left(h^{*}, p^{*}\right)$, de acordo com as condições acima, para $\gamma=0,9$ (considerando que a cada geração sobrevivem $90 \%$ dos predadores).

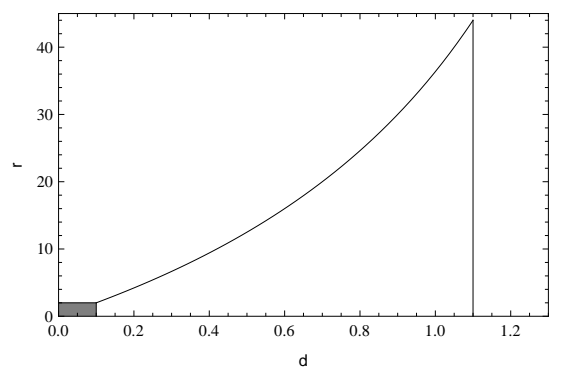

Figura 1: Região de estabilidade dos pontos de equilíbrio $\left(h_{1}, p_{1}\right)$, em cinza, e $\left(h^{*}, p^{*}\right)$, para $\gamma=0,9$.

Passamos agora a considerar escalas de tempo diferentes para a reprodução das duas espécies. Introduziremos mais um parâmetro: N - inteiro e positivo. Analisaremos as duas situações:

Caso 1: a presa se reproduz $N$ vezes no intervalo de tempo no qual o predador se reproduz uma vez (presa rápida - predador lento) 


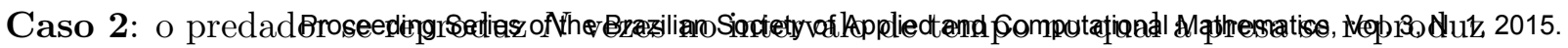
uma vez (predador rápido - presa lenta)

Consideramos como geração padrão do sistema aquela em que ocorre a reprodução da espécie lenta e supomos gerações intermediárias nas quais ocorre a reprodução da espécie rápida.

No caso 1 (presa rápida - predador lento) temos a cada geração intermediária: diminuição das presas devido a predação; reprodução das presas; e diminuição da densidade de predadores por mortalidade natural. A cada geração padrão temos todos os eventos que ocorrem nas gerações intermediárias mais a reprodução dos predadores (proporcionalmente à quantidade média de presas predada por geração intermediária).

Assim, sendo $h_{t}$ e $p_{t}$, respectivamente, as densidades adimensionais de presas e predadores na geração $t$, as equações adimensionais que descrevem a dinâmica do caso 1 são:

$$
\left\{\begin{array}{l}
h_{t+1}=f_{h}\left(F^{N-1}\left(h_{t}, p_{t}\right)\right) \\
p_{t+1}=\gamma^{N} p_{t}+d \gamma^{N-1} p_{t} \frac{1}{N}\left(h_{t}+f_{h}\left(h_{t}, p_{t}\right)+f_{h}\left(F\left(h_{t}, p_{t}\right)\right)+\ldots+f_{h}\left(F^{N-2}\left(h_{t}, p_{t}\right)\right)\right)
\end{array}\right.
$$

onde

$$
\begin{aligned}
F & : \mathbb{R}^{2} \longrightarrow \mathbb{R}^{2} \\
F(h, p) & =\left(f_{h}(h, p), f_{p}(h, p)\right)=\left(h e^{r(1-h-p)}, \gamma p\right) .
\end{aligned}
$$

$F^{N-1}$ representa a função $F$ composta $N-1$ vezes. Quando $N=1$, temos o modelo com uma única escala de tempo dado em (1).

Já no caso 2 (presa lenta - predador rápido) temos a cada geração intermediária: diminuição das presas devido a predação; crescimento dos predadores segundo um fator dependente da densidade de presas; e diminuição da densidade de predadores por mortalidade natural. A cada geração padrão temos todos os eventos que ocorrem nas gerações intermediárias mais a reprodução das presas.

Assim, sendo $h_{t}$ e $p_{t}$, respectivamente, as densidades adimensionais de presas e predadores na geração $t$, as equações adimensionais que descrevem a dinâmica do caso 2 são:

$$
\left\{\begin{array}{l}
h_{t+1}=g_{h}\left(G^{N-1}\left(h_{t}, p_{t}\right)\right) \\
p_{t+1}=g_{p}\left(G^{N-1}\left(h_{t}, p_{t}\right)\right)
\end{array}\right.
$$

onde

$$
\begin{aligned}
G & : \mathbb{R}^{2} \longrightarrow \mathbb{R}^{2} \\
G(h, p) & =\left(h e^{-r p}, \gamma p+d h p\right),
\end{aligned}
$$

$\mathrm{e}$

$$
\begin{aligned}
g_{h}, g_{p} & : \mathbb{R}^{2} \longrightarrow \mathbb{R} \\
g_{h}(h, p) & =h e^{r(1-h-p)} \\
g_{p}(h, p) & =\gamma p+d h p
\end{aligned}
$$

$G^{N-1}$ representa a função $G$ composta $N-1$ vezes. Quando $N=1$, temos o modelo com uma única escala de tempo dado em (1).

A função $G$ na Equação (5) descreve o que ocorre em cada geração intermediária e as funções $g_{h}$ e $g_{p}$ descrevem o que ocorre em cada geração padrão.

\section{Simulações}

Todas as simulações apresentadas neste trabalho consideram $\gamma=\sqrt[N]{0,9}$, onde $N$ é o parâmetro para múltiplas escalas já definido anteriormente, assim a cada geração (padrão) temos a sobrevivência de $90 \%$ dos predadores. Foram realizadas simulações para outros valores de $\gamma$, mas não foi percebida mudança significativa no tamanho da região de estabilidade do equilíbrio de coexistência das espécies. 


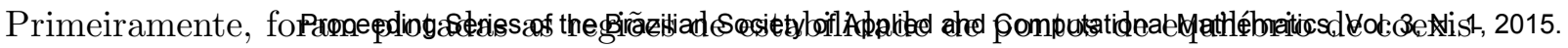
tência das espécies, em função dos parâmetros $r$ e $d$. Estes gráficos foram construídos plotando-se os pontos $(d, r)$, para os quais ambos os autovalores da matriz jacobiana do modelo calculada no ponto de equilíbrio de coexistência tinham valor absoluto menor do que um.

(a)

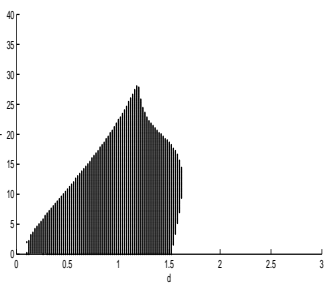

(b)

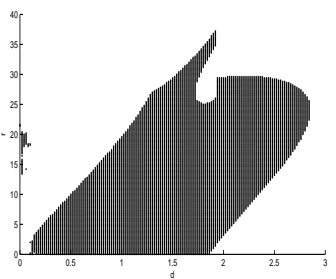

Figura 2: Região de estabilidade para o caso 1 com $N=2$ (a) e 4 (b).

(a)

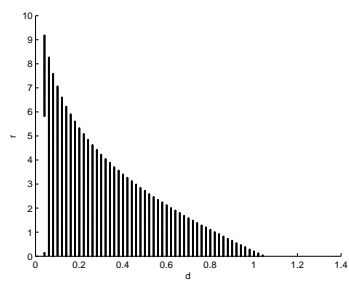

(b)

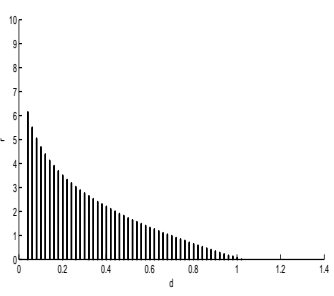

Figura 3: Região de estabilidade para o caso 2 com $N=2$ (a) e 4 (b).

Comparando as Figuras 2 (caso 1) e 3 (caso 2) com a região de estabilidade do modelo original (Figura 1), podemos observar que a consideração de múltiplas escalas de tempo para a reprodução das espécies modifica a região de estabilidade do equilíbrio de coexistência das espécies. A dinâmica vital da presa mais rápida do que a do predador levaria um aumento dessa região, enquanto que a hipótese contrária levaria a uma diminuição.

Passamos então a analisar as densidades de equilíbrio de presas e predadores. Na Figura 4 são plotadas essas densidades para ambos os casos, com valores dos parâmetros para os quais temos equilíbrio estável para todos os valores de $N(r=1,8$ e $d=0,11)$. As densidades foram analisadas para vários outros valores para os parâmetros $d$ e $r(r \leq 5)$ tomados dentro da região de estabilidade de pontos de equilíbrio de coexistência e este comportamento foi semelhante. Observa-se aumento na densidade de presas quando estas possuem dinâmica mais rápida e diminuição quando possuem dinâmica mais lenta. Isto estaria de acordo com a hipótese de Kindlmann e Dixon [2] mencionada anteriormente.

(a)

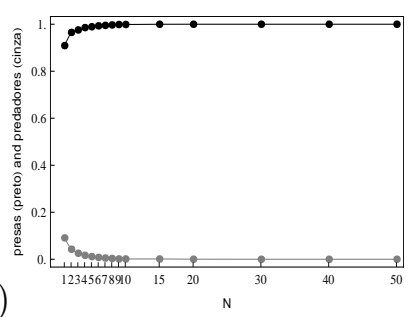

(b)

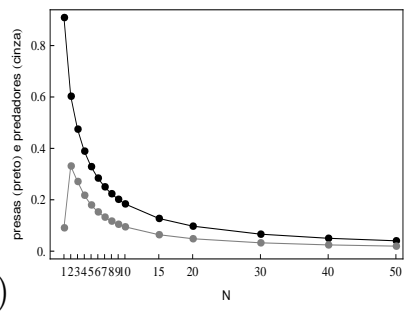

Figura 4: Densidades de presas (em preto) e predadores (em cinza) em função de $N$ para o caso 1 - presa rápida (a) e caso $\mathbf{2}$ - predador rápido (b).

\section{Modelos com estrutura espacial}




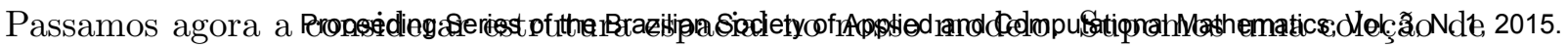
sítios enumerados por $1,2, \ldots, n$ e em cada um desses sítios existe uma comunidade de duas espécies (presa e predador) que chamamos população local ou subpopulação. Denotamos por $h_{t}^{j}$ e $p_{t}^{j}$ a densidade de presas e predadores, respectivamente, no sítio $j$, no tempo $t$. Estabelecemos conexões entre os sítios, ou seja, a possibilidade dos indivíduos migrarem para outros sítios. A topologia da rede utilizada será descrita por uma matriz $C=\left[c_{j i}\right]_{n \times n}$, em que os elementos $c_{j i}$ representam a proporção de indivíduos que migra do sítio $i$ para o sítio $j$. Os parâmetros $\mu_{h} \mathrm{e}$ $\mu_{p}$ representam a proporção de presas e predadores, respectivamente, que deixa cada sítio, logo $0 \leq \mu_{h} \leq 1$ e $0 \leq \mu_{p} \leq 1$

Consideraremos que a migração ocorre de forma coerente com a escala de reprodução, ou seja, a espécie que se reproduz nas gerações intermediárias, também migrará nessas gerações, enquanto que a espécie lenta, que só se reproduz nas gerações padrões, só migrará nessas gerações. Assim, a cada intervalo de reprodução (e migração) da espécie lenta, a espécie rápida se reproduzirá e migrará $N$ vezes.

No caso 1, então, suporemos as presas se reproduzindo e migrando nas gerações intermediárias, enquanto que os predadores só se reproduzirão e migrarão nas gerações padrões. Assim, a dinâmica metapopulacional do caso 1 será descrita pelos seguintes sistemas:

Nas gerações intermediárias, para $k=0,1, \ldots, N-2$, temos

$$
\begin{aligned}
h_{t, k+1}^{j} & =\left(1-\mu_{h}\right)\left(h_{t, k}^{j} e^{r\left(1-h_{t, k}^{j}-p_{t, k}^{j}\right)}\right)+\sum_{i=1}^{n} c_{j i} \mu_{h}\left(h_{t, k}^{i} e^{r\left(1-h_{t, k}^{i}-p_{t, k}^{i}\right)}\right) \\
p_{t, k+1}^{j} & =\gamma p_{t, k}^{j}
\end{aligned}
$$

Na geração padrão, temos

$$
\begin{aligned}
h_{t+1}^{j}= & \left(1-\mu_{h}\right)\left(h_{t, N-1}^{j} e^{r\left(1-h_{t, N-1}^{j}-p_{t, N-1}^{j}\right)}\right)+\sum_{i=1}^{n} c_{j i} \mu_{h}\left(h_{t, N-1}^{i} e^{r\left(1-h_{t, N-1}^{i}-p_{t, N-1}^{i}\right)}\right) \\
p_{t+1}^{j}= & \left(1-\mu_{p}\right)\left(\gamma^{N} p_{t}^{j}+d \gamma^{N-1} p_{t}^{j} \frac{1}{N}\left(h_{t}^{j}+h_{t, 1}^{j}+h_{t, 2}^{j}+\ldots+h_{t, N-1}^{j}\right)\right) \\
& +\sum_{i=1}^{n} c_{j i} \mu_{p}\left(\gamma^{N} p_{t}^{i}+d \gamma^{N-1} p_{t}^{i} \frac{1}{N}\left(h_{t}^{i}+h_{t, 1}^{i}+h_{t, 2}^{i}+\ldots+h_{t, N-1}^{i}\right)\right) .
\end{aligned}
$$

Já no modelo para o caso $\mathbf{2}$, teremos predadores se reproduzindo e migrando nas gerações intermediárias e presas apenas nas gerações padrões. Essa dinâmica será descrita então pelos sistemas:

Nas gerações intermediárias, para $k=0,1, \ldots, N-2$, temos:

$$
\begin{aligned}
h_{t, k+1}^{j} & =h_{t, k}^{j} e^{-r p_{t, k}^{j}} \\
p_{t, k+1}^{j} & =\left(1-\mu_{p}\right)\left(\gamma p_{t, k}^{j}+d h_{t, k}^{j} p_{t, k}^{j}\right)+\sum_{i=1}^{n} c_{j i} \mu_{p}\left(\gamma p_{t, k}^{i}+d h_{t, k}^{i} p_{t, k}^{i}\right)
\end{aligned}
$$

Na geração padrão, temos

$$
\begin{aligned}
& h_{t+1}^{j}=\left(1-\mu_{h}\right)\left(h_{t, N-1}^{j} e^{r\left(1-h_{t, N-1}^{j}-p_{t, N-1}^{j}\right)}\right)+\sum_{i=1}^{n} c_{j i} \mu_{h}\left(h_{t, N-1}^{i} e^{r\left(1-h_{t, N-1}^{i}-p_{t, N-1}^{i}\right)}\right) \\
& p_{t+1}^{j}=\left(1-\mu_{p}\right)\left(\gamma p_{t, N-1}^{j}+d h_{t, N-1}^{j} p_{t, N-1}^{j}\right)+\sum_{i=1}^{n} c_{j i} \mu_{p}\left(\gamma p_{t, N-1}^{i}+d h_{t, N-1}^{i} p_{t, N-1}^{i}\right)
\end{aligned}
$$

Supondo que $\sum_{i=1}^{n} c_{j i}=1$ para todo $j=1,2, \ldots, n$, podemos mostrar que $X=\left(\mathrm{x}^{*}, \mathrm{x}^{*}, \ldots, \mathrm{x}^{*}\right)$, em que $\mathrm{x}^{*}=\left(h^{*}, p^{*}\right)$ é o ponto de equilíbrio da dinâmica local (1), é um ponto de equilíbrio dos sistemas dos casos 1 e 2 acima. 


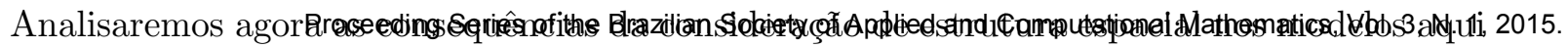
estudados. Nas simulações suporemos 30 sítios e duas matrizes de configuração: local e global. No caso da matriz de configuração local, supomos um anel de $n$ sítios simetricamente acoplados com os dois vizinhos mais próximos, assim a matriz de configuração $C$ é uma matriz circulante

$$
C=\operatorname{circ}\left(0, \frac{1}{2}, 0, \ldots, 0, \frac{1}{2}\right) \text {. }
$$

Já no caso da matriz de configuração global, a topologia da rede utilizada é um anel de $n$ sítios simetricamente acoplados com todos os vizinhos, assim a matriz de configuração $C$ é a matriz circulante

$$
C=\operatorname{circ}\left(0, \frac{1}{n-1}, \frac{1}{n-1}, \ldots, \frac{1}{n-1}\right)
$$

Passamos, então, a analisar a instabilidade causada pela migração. Consideraremos valores dos parâmetros para os quais a dinâmica local era estável e plotaremos regiões dos parâmetros de migração para os quais teremos o sistema com estrutura espacial instável.

Foram plotadas as regiões de instabilidade apenas para $N=2$ devido à complexidade do sistema. A verificação da estabilidade de $X=\left(\mathrm{x}^{*}, \mathrm{x}^{*}, \ldots, \mathrm{x}^{*}\right)$ envolve o cálculo de uma matriz jacobiana de dimensões 60x60. A Figura 5 traz uma dessas regiões para o caso 1 e matriz de configuração local. Podemos observar que as instabilidades ocorrem quando o coeficiente de difusão das presas é grande combinado com coeficiente do predador sendo pequeno ou bastante grande, diferentemente da instabilidade provocada pela migração no modelo original que ocorre apenas para valores assimétricos dos coeficientes de migração. Quando temos a instabilidade do equilíbrio $X=\left(\mathrm{x}^{*}, \mathrm{x}^{*}, \ldots, \mathrm{x}^{*}\right)$ o sistema apresenta distintos equilíbrios estáveis entre os sítios, soluções caóticas, soluções periódicas e quase-periódicas. Exceto para os casos de soluções caóticas, quando a migração desestabiliza a dinâmica temos redução da densidade média de presas e aumento da densidade média de predadores. As maiores regiões de instabilidade difusiva ocorrem para valores grandes de $r$ e para a matriz de configuração local.

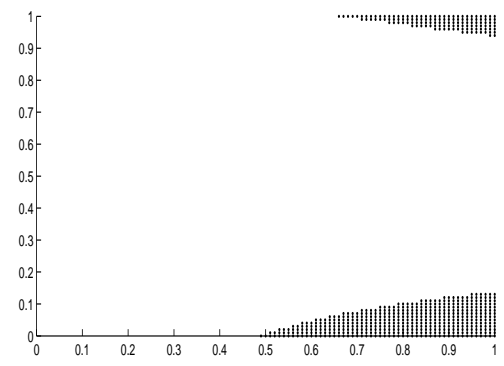

Figura 5: Região de instabilidade difusiva para caso 1 , com $N=2, r=5, d=1$ e matriz de configuração local.

Já para o caso 2, ocorrem bem menos instabilidades causadas pela migração (comparandose com o caso 1 e até mesmo com o modelo original, com uma única escala de tempo). $\mathrm{X}^{*}=$ $\left(\mathrm{x}^{*}, \mathrm{x}^{*}, \ldots, \mathrm{x}^{*}\right)$ apenas torna-se instável para valores do coeficiente de migração dos predadores bastante baixos, em torno de 0,01. Nesse caso, temos densidades constantes para uma quantidade de gerações suficientemente grande em cada um dos sítios, mas estes valores são distintos de um sítio para o outro. Quando analisado o efeito da estrutura espacial sobre dinâmicas instáveis, observamos um efeito estabilizante da migração a partir de determinados valores do coeficiente de migração dos predadores. Estes valores ficam em torno de $\mu_{p}=0,1$ até $\mu_{p}=0,55$, variando conforme os valores de $r$ e $d$ fixados. A Figura 6 traz esses valores em função do parâmetro $d$ para $r$ fixado em 4, em que verificamos que quanto maior for o parâmetro $d$ (associado a taxa de conversão de presas em predadores) maior precisará ser o coeficiente de migração dos predadores para que a dinâmica torne-se estável. 


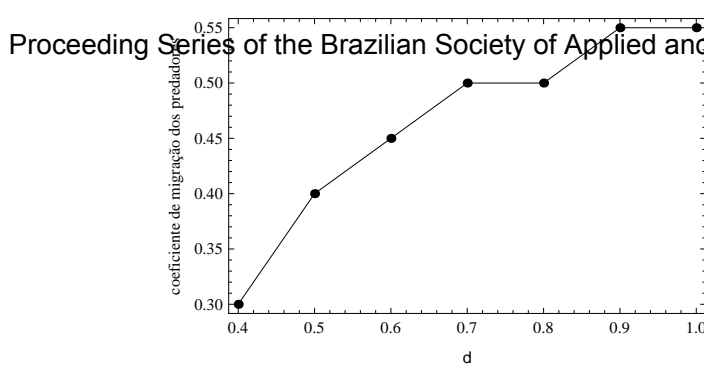

Figura 6: Valores do parâmetro de migração dos predadores a partir do qual a dinâmica instável localmente torna-se estável, caso $2, N=2$ e $r=4$.

\section{Conclusões}

O presente trabalho propôs um modelo presa-predador com múltiplas escalas de tempo para dinâmica vital e migração das duas espécies. Na análise da dinâmica local observou-se uma modificação da região de estabilidade do equilíbrio de coexistência em relação ao modelo com uma única escala. Tivemos um aumento na estabilidade do modelo em que a presa é a espécie mais rápida (caso 1) e diminuição no outro caso. Também pudemos observar aumento na densidade de presas no caso 1 e diminuição no caso 2, vindo ao encontro dos estudos de Kindlmann et al. [3]. Finalmente, considerou-se o modelo com estrutura espacial e migração coerente com a escala de reprodução. Em ambos os casos, percebe-se maior influência do coeficiente de migração da espécie rápida. O modelo presa rápida-predador lento (caso 1) mostrou-se mais suscetível a instabilidades causadas pela migração do que o modelo predador rápido (caso 2), o qual apresenta maior estabilidade.

\section{Referências}

[1] C. L. Andrade, "Múltiplas Escalas em Interações Presa-Predador", Dissertação de Mestrado, PPGMat - UFSM, 2010.

[2] P. Kindlmann, A. F.G. Dixon, Generation Time Ratios - Determinants of Prey Abundance in Insect Predator-Prey Interactions, Biological Control, 16 (1999) 133-138.

[3] P. Kindlmann, H. Yasuda, Y. Kajita,A. F. Dixon, Field Test of the Effectiveness of Ladybirds in Controlling Aphids, em "International Symposium on Biological Control of Arthropods" pp. 441-447, Davos, 2005.

[4] W. W. Murdoch, C. J. Briggs, S. Swarbrick, Host supression and stability in a parasitoid-host system: experimental demonstration, Science, 309 (2005) 610-613.

[5] M. G. Neubert, M. Kot, The Subcritical Collapse of Predator Population in Discrete-Time Predator-Prey Models, Mathematical Biosciences, 110 (1992) 45-66.

[6] E. Pachepsky, R. M. Nisbet, W. W. Murdoch, Between discrete and continuous: consumerresource dynamics with synchronized reproduction, Ecology, 89 (2008) 280-288.

[7] L. A. D. Rodrigues, D. C. Mistro, C. L. Andrade, Sistema Presa-Predador com Duas Escalas de Crescimento: Presa Rápida x Predador Lento, Biomatemática, 20 (2010) 69-78.

[8] P. Rohani, G. D. Ruxton, Dispersal-Induced Instabilities in Host-Parasitoid Metapopulations. Theoretical Population Biology, 55 (1999) 23-36.

[9] A. M. Turing, The Chemical Basis of Morphogenesis. Phil. Trans. Roy. Soc. Lond., 237B (1952) 37-72. 